\title{
Palinacousis-Auditory Perseveration
}

\section{Case Report}

Journal of Epilepsy Research pISSN 2233-6249 / eISSN 2233-6257

Received April 10, 2017

Accepted May 17, 2017

Corresponding author: Kwang-Ki Kim Department of Neurology, Dongguk University Ilsan Hospital, 27 Dongguk-ro, Ilsandong gu, Goyang 10326, Korea

Tel. +82-31-961-7200

Fax. +82-31-961-7212

E-mail; 70neukim@naver.com

\section{Soo-Hyun Park, Kwang-Ki Kim}

Department of Neurology, Dongguk University Ilsan Hospital, Goyang, Korea
Palinacousis is an auditory illusion rarely reported in cases of temporal lobe dysfunction. After reviewing the literature and comparing our clinical experience, we believe that palinacousis can occur as an aura, a simple partial seizure, a complex partial seizure, and/or a postictal event. Palinacousis maybe more common than recognized in patients with receptive aphasias or diffuse cerebral dysfunction, whose language deficits preclude adequate description. We report the case of a 71 years old man with the previously diagnosis of an infarction of left middle cerebral artery and valvular heart disease who presented with the symptoms of palinacousis. He was not diagnosed psychotic diseases. An electroencephalography showed normal wave. He was diagnosed as temporal lobe epilepsy with palinacousis due to post-stroke. Palinacousis was completely reversed after treatment with antiepileptic drug. We report that palinacousis as an epileptic phenomenon can help localized potential lesions and can improve patient care. (2017;7:57-59)

Key words: Palinacousis, Post-stroke seizure, Temporal lobe epilepsy, Electroencephalography

\section{Introduction}

Palinacousis was described as a paroxysmal auditory illusion in $1971^{1}$ that was derived from the Greek words palin, which means again, and acousis, meaning hearing. ${ }^{2}$ Palinacousis is auditory phenomenon produced by environmental aural stimulus, and persist or recur after the initial acoustic stimulus has stopped. ${ }^{3}$ It is important that distinguish palinacousis from other types of auditory hallucinations which occur in psychiatric disease. For example, palinacousis is triggered by an external auditory stimulus, whereas psychiatric hallucinations often occur thought echoing without actual sensory trigger. $^{4}$

The lesions that cause palinacousis are variable (ie, tumors, vascular malformations, and intracerebral hemorrhages). Also, there are various mechanisms that contribute to the palinacousis. ${ }^{1}$ There is a strong hypothesis that palinacousis occurs in the auditory cortex of the dominant temporal lobe, temporoparietal junction, or temporooccipital junction. ${ }^{5}$ We report the case of palinacousis with temporal lobe epilepsy after stroke.

\section{Case}

A 71-year-old right-handed man was admitted to our hospital.
When he came to the hospital, he was in a state of aphasic seizure with repeated spitting. He was diagnosed as an infarction of left middle cerebral artery territory with valvular heart disease 20 years ago. He was right side weakness (mRS score 1) with a sequelae of cerebral infarction. Aphasic seizure is the secondary event after he was diagnosed with cerebral infarction. He initially presented with an aphasic seizure for 5 years ago. He was treated with valproic acid, which was discontinued due to poor cooperation.

On neurological examination, he was an alert mentality. Speech was not fluent tangentially with improper comprehension. He made frequent paraphasic errors. He was not able to repeat a short phrase and name. Pupils were reactive, extraocular movements were conjugate. He had right side weakness (MRC grade 4) and right central type facial palsy due to previous cerebral infarction.

After arriving to the hospital, he was found to have a left parietal cerebromalatic change due to cerebral infarction by non-contrast brain CT. There were no new lesions associated with symptoms in brain magnetic resonance imaging (MRI). Brain MRI on admission showed only cerebromalatic lesions in left temporo-parietal lobe due to previous cerebral infarction (Fig.1). ${ }^{6}$ His symptoms of apparent motor aphasia were thought to be a temporal lobe epilepsy due to post-stroke and he was started on fosphenytoin with loading dose. On the second day of admition, aphasia resolved. 
But, as him aphasia improved, the patient reported a new auditory phenomenon after hearing a dialogue on television. He was not an aura preceding his symptoms. He stated that a dialogue rattled around in his head and repeated itself numerous times. Acoustic sensations preserved after the television was switched off. He could not stop the dialogue by manually occluding his external auditory meatus. He had never experienced auditory illusions or hallucinations in the past and had no history of psychiatric illness. The nature of his auditory perseveration (palinacousis) did not accompany with motor aphasia. He said the episode was bothersome and distracting symptoms.

At the time of symptoms, an electroencephalogram (EEG) revealed

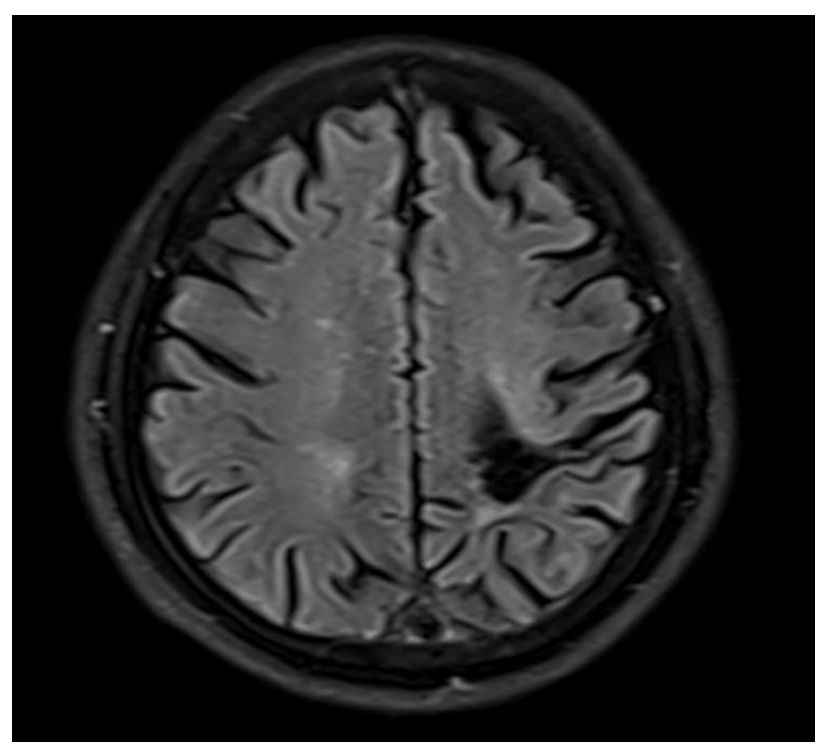

Figure 1. Brain MRI (T2 Flair) presented a large geographic cerebromalatic change in left parietal lobe. MRI, magnetic resonance imaging.

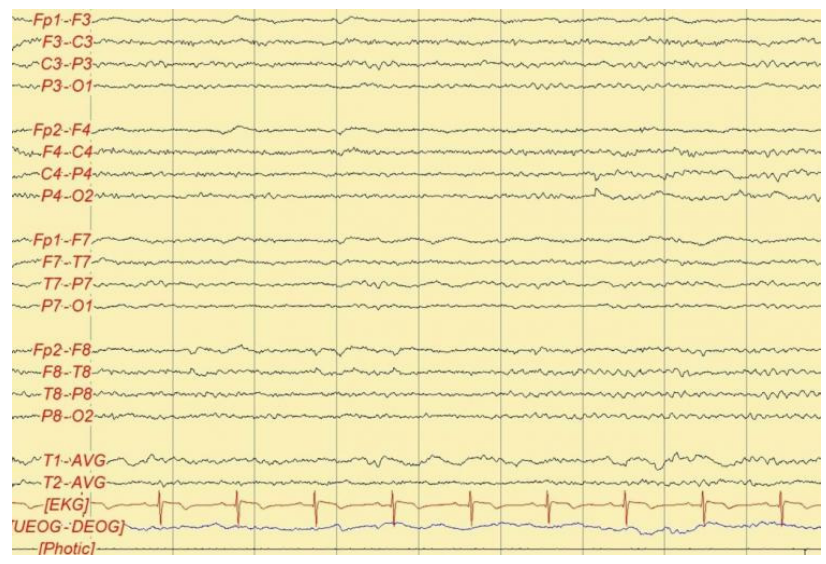

Figure 2. An electroencephalography (EEG) presented without epileptiform abnormalities. without definite epileptiform abnormalities (Fig. 2). Brain perfusion MRI showed decreased perfusion in old lesion with suspicious increased perfusion at overlying cortex due to seizure related hyperperfusion (Fig. 3). We concerned that the patients symptoms were focal sensory seizures, and prescribed antiepileptic drug with levetiracetam $250 \mathrm{mg}$ twice a day continuous. After giving antiepileptic drug, he presented the perseverating acoustic phenomenon were reduced. By hospital day 4, palinacousis and motor aphasia had completely resolved.

\section{Discussion}

Palinacousis is an auditory illusion that external auditory stimuli are perseverated paroxysmal. ${ }^{1}$ And then, auditory illusion can last from several seconds to several hours. According to previous reports, symptoms of organic brain disease such as epilepsy, malignancy, hemorrhage, ${ }^{7}$ traumatic and infection, it must to be differentiated from auditory hallucinations in psychiatric disease as well as psychotic symptoms seen postictally. ${ }^{8}$ There have been case reports of misdiagnosed schizophrenics as well as schizophrenics with palinacousis secondary to a temporal lobe seizure focus. ${ }^{9}$ Because of the rare occurrence of palinacousis, it can easily be misdiagnosed with a psychiatric disorder, especially if patients have a past history of psychiatric disorder. Thought echoing which are characteristic of schizophrenia are not palinacoustic phenomena because there is no actual

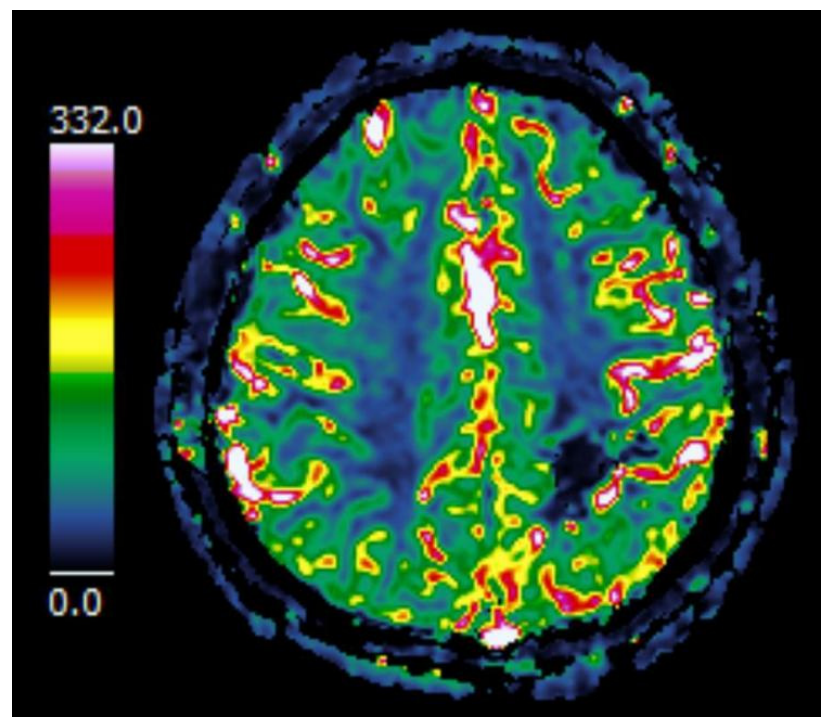

Figure 3. Brain perfusion magnetic resonance imaging (MRI) showed increased perfusion at overlying cortex due to seizure related hyperperfusion. 
Soo-Hyun Park, et al. Palinacousis in post-stroke seizure 59

external auditory irritation. ${ }^{10}$ Auditory hallucinations of psychiatric disorders rarely occurred environmentally evoked sound. Psychotic patients are also highly personalized and seldom neutral in content. ${ }^{4}$ Palinacousis appear immediately after the initial stimulus. Palinacousis is episodic, and not find in psychotic or neurotic behavior. $^{4}$

The mechanism underlying palinacousis remains unclear but the available evidence strongly suggests that it is an epileptic phenomenon. ${ }^{5}$ Jacobs et al. ${ }^{1}$ regarded palinacousis as clinical manifestation of temporal lobe seizure activity. There is a strong association with seizures reported in the literature. ${ }^{11}$ A postictal mechanism has been proposed a loss of normal suppression of auditory perseveration. That is, palinacousis is a negative release phenomenon resulting from loss of function of normally suppressed auditory perseveration in auditory cortex. In previous reports, most patients had lesions involving the temporal lobes, associated with the auditory cortex. ${ }^{12}$ In our patients, brain image showed organic changes in the left tempo-parietal region. Palinacousis is related to transient hyperexcitation of supplementary auditory pathways.

Palinacousis is rare, though it may be more common than recognized for a variety of reasons. After reviewing the case reports, palinacousis can occur in a variety of settings; either or not as an aura, focal sensory seizure, generalized motor seizure, or as a postictal event.

\section{References}

1. Jacobs L, Feldman M, Diamond SP, Bender MB. Palinacousis: persistent or recurring auditorysensations. Cortex 1973;9:275-87.

2. Fields MC, Marcuse LV. Palinacousis. Handb Clin Neurol 2015;129: 457-67.

3. Bega $D$, Wang $N$, Klein JP. Reversible Palinacousis From Intracranial Metastases. Neurohospitalist 2014;4:22-5.

4. Prueter $C$, Waberski TD, Norra $C$, et al. Palinacousis leading to the diagnosis of temporal lobe seizures in a patient with schizophrenia. Seizure 2002;11:198-200.

5. Di Dio AS, Fields MC, Rowan AJ. Palinacousis - auditory perseveration: two cases and a review of the literature. Epilepsia 2007;48: 1801-6.

6. Szabo K, Poepel A, Pohlmann-Eden B, et al. Diffusion-weighted and perfusion MRI demonstrates parenchymal changes in complex partial status epilepticus. Brain 2005;128:1369-76.

7. Patterson MC, Tomlinson FH, Stuart GG. Palinacousis: a case report. Neurosurger 1988;22:1088-90.

8. Kim JS, Kwon M, Jung JM. Palinacousis in temporal lobe intracerebral hemorrhage. Neurology 2007;68:1321-2.

9. Differential diagnosis of palinacousis in a psychiatric patient. Am J Psychiatry 1983;140:1067-8.

10. Terao T, Matsunaga K. Musical hallucinations and palinacousis. Psychopathology 1999;32:57-9.

11. Mohamed W, Ahuja N, Shah A. Palinacousis--evidence to suggest a post-ictal phenomenon. J Neurol Sci 2012;317:6-12.

12. Penfield $W$, Perot $P$. The brain's record of auditory and visual experience. A final summary and discussion. Brain 1963;86:595-696. 